UDC: 821.134.2(72).09 Fuentes C.

821.134.2.09 Goytisolo J.

DOI: https://doi.org/10.18485/hispserb.2019.2.ch18

\author{
Isidora Kalović ${ }^{1}$ \\ Doctoranda en la Universidad de Belgrado \\ Serbia
}

\title{
PROCEDIMIENTOS NARRATIVOS DE CARLOS FUENTES Y JUAN GOYTISOLO
}

\section{Resumen}

En este trabajo analizamos los procedimientos narrativos en las novelas La muerte de Artemio Cruz de Carlos Fuentes y Señas de identidad de Juan Goytisolo. Comparamos los procedimientos narrativos de Fuentes y Goytisolo analizando estructuras narrativas de las novelas, técnicas narrativas, temporalidad, narrador y focalización. Comentamos estos aspectos de las novelas porque representan aspectos fundamentales a la hora de hablar sobre la narración, además, ambos escritores demostraron que la estructura, el lenguaje y la técnica son tan importantes para su creación como la historia misma. Considerando que tratamos un tema comparativo, buscamos y describimos las características comunes de los procedimientos narrativos, pero también señalamos las diferencias. Veremos que tanto el tratamiento de la novela y del papel de escritor como la estrategia narrativa de Fuentes y Goytisolo son similares, y podemos decir que lo dicho representa el encuentro de la novela española con la hispanoamericana en los años sesenta del siglo XX. Aunque sería posible encontrar otras características y muchos otros ejemplos analizando detalladamente cada capítulo, trataremos solamente los más llamativos para ilustrar los fenómenos que primero describimos o definimos teóricamente.

Palabras clave: Carlos Fuentes, Juan Goytisolo, procedimiento narrativo, novela, narración.

\footnotetext{
${ }^{1}$ isidorakalovic@gmail.com
} 


\section{Introducción}

Dos contemporáneos, escritores y amigos, Carlos Fuentes y Juan Goytisolo, compartieron sus puntos de vista sobre diferentes temas literarios y en sus obras elaboraron temas similares. Según Fuentes (2013), Goytisolo representa el encuentro de la novela española con la que se escribe en América Hispana, mientras Juan Goytisolo cree que la obra de Fuentes representa una "enciclopedia de especie humana" (Goytisolo 2011). Ambos escritores buscan la identidad, tanto personal como la identidad de toda la nación o país, Carlos Fuentes de México, Juan Goytisolo de España. Ambos consideran que una cultura es el producto de todos los factores que han influido en ella. Además, los dos comprenden la identidad de un individuo, una nación y una cultura a través del pasado, el presente y el futuro. Goytisolo cita la observación de Bajtín de que una obra literaria debe basarse en el pasado, porque si solo vive en el presente, desaparece con él (Goytisolo 2011). Esta tendencia es también un rasgo similar en las obras de Fuentes y Goytisolo.

En este artículo analizamos las novelas La muerte de Artemio Cruz de Carlos Fuentes del año 1962 y Señas de identidad de Juan Goytisolo de 1966. Fuentes y Goytisolo eligen la novela como género literario adecuado para la búsqueda de la identidad personal y nacional. Ellos ponen en el primer plano la experimentación con el lenguaje, la estructura y la forma, que se convierte en un rasgo más importante que la historia narrada (Stecher 2002: 68). Además del tratamiento común del tema de la identidad nacional, las técnicas narrativas y los procedimientos narrativos tienen muchas similitudes. La narración, según Rimmon-Kenan (2006: 7), es el término que abarca el proceso de comunicación en el que la narración se envía como un mensaje por un lado, y el carácter verbal de los medios a través de los cuales se transmite un mensaje, por el otro. Por lo tanto, el analisis de la narración y sus aspectos formales principales como la estructura, la técnica narrativa, la temporalidad, la focalización y el narrador, es muy importante. Especialmente cuando se trata de la obra de Fuentes y Goytisolo que cuidadosamente eligen sus procedimientos narrativos para que la narración entera produzca el efecto deseado.

\section{Estructura narrativa}

El escritor latinoamericano de la nueva novela hispanoamericana sintió la necesidad de cambiar su lengua para compensar todo lo que, según Carlos Fuentes, la historia ha silenciado. Esta revolución literaria 
crea el lenguaje de la polisemia, alusión y apertura (Fuentes 2013). Las soluciones artísticas que ofrece la nueva novela hispanoamericana llevan consigo diferentes novedades en las estructuras narrativas de las novelas propias. Este es también el caso de la estructura de la novela La muerte de Artemio Cruz, que al principio nos confunde con las diferentes unidades narrativas ${ }^{2}$, después de una lectura continuada comprendemos que hay una estructuración en la estructura narrativa, que a primera vista parece caótica. Dentro de la novela no hay división en partes o capítulos, pero claramente se distinguen unidades o tres niveles narrativos. La primera es la narración en primera persona, yo, la segunda consiste de la narración en segunda persona, tú, la tercera, consiste de la narración en tercera persona, él, y solamente ella lleva algún tipo de título, que es la fecha en que la cual sucede lo narrado en esta unidad. Después de leer la novela, notamos que está realmente muy bien estructurada, las secuencias yo, tú, él, van en este orden, doce veces, y al final de la novela, una vez más, aparecen las unidades yo y tú. En los últimos fragmentos de las secuencias yo y él, el protagonista fallece, de este modo la entidad retrospectiva no aparece por decimotercera vez. Después de leer la segunda serie de estas unidades narrativas, el lector ya no se sorprende, y sabe muy bien qué unidad sigue. Al final, vemos que aunque los pronombres personales difieren, los tres representan a Artemio Cruz:

Yo no sé... no sé... si él soy yo... si tú fue él... si yo soy los tres... Tú... te traigo dentro de mí y vas a morir conmigo... Dios... Él... lo traje adentro y va a morir conmigo... los tres... que hablaron... Yo... lo traeré adentro y morirá conmigo... sólo... (Fuentes 2008: 358)

La trama narrativa en la que el personaje principal se encuentra en un estado modificado de consciencia, a punto de morir, explica y justifica esta estructura (González Boixo 2001: 28). Está de acuerdo con la consciencia parcial de Artemio Cruz y los recuerdos que aparecen en su mente. Cada unidad es narrada en diferente tiempo verbal, de modo que lo que se dice en la unidad yo representa la actualidad: "Yo no sé..." (Fuentes 2008: 358), en la unidad narrativa tú, predomina el futuro: "Tú ya no sabrás..." (Fuentes 2008: 358), mientras que los eventos de la secuencia él, están en el tiempo pasado: "él lloró y empezó a vivir..." (Fuentes 2008: 358). Justamente esta elección de tiempos verbales muestra la percepción de Fuentes sobre la identidad humana y nacional, la integridad del hombre

\footnotetext{
${ }^{2}$ González Boixo las llama también secuencias. Este el término que nosotros utilizaremos en el trabajo.
} 
y la nación se encuentran en la trinidad de su pasado, presente y futuro. Artemio Cruz lleva sus yo, tú y él, es decir, su pasado, presente y futuro, todo esto forma parte de él y finalmente muere con él, es inseparable de su pasado, presente y futuro. Sin embargo, esta trinidad no se refleja solamente en la presentación de Artemio en su juventud, madurez y viejez, sino que también se puede reflejar en la presentación de tres posiciones económicas y sociales diferentes a lo largo de su vida, en su vida del antes, durante y después de la Revolución Mexicana, en un conflicto familiar, también, que se divide en tres direcciones, con su esposa, su hija y su nieto, y al final su apellido, que denota una cruz, representa un símbolo de la Trinidad cristiana (Blanc 1988: 88, 92). Como observa Blanc (1988: 92), Fuentes elige cuidadosamente este apellido para su héroe, porque la cruz se representa con dos líneas, la vertical cruza la horizontal y, por otro lado, simboliza la trinidad. Este aspecto dual es el segundo aspecto de la novela y se relaciona con la dualidad asociada con la cultura azteca y se manifiesta en la novela en la representación de extremidades como la vida y la muerte; tal vez la imagen más llamativa se encuentra en las últimas páginas de la novela donde se presenta el nacimiento de Cruz en el año 1889, e inmediatamente después su muerte en 1959. La dualidad también está presentada a través de la relación entre el amor y el odio, y cuando se trata de la sintaxis, aparece la repetición de palabras que a menudo se presentan son como si estuvieran en un espejo. Artemio Cruz es como la novela misma, una mezcla de estos dos aspectos. Su identidad e identidad de México representan una mezcla inseparable de dos razas, dos culturas, culturas de dualidad y trinidad, azteca y cristiana. Podemos concluir que la estructura también tiene los rasgos de dualidad y trinidad al mismo tiempo, porque, por un lado, hay unidades narrativas sin nombre, sin título, y por otro, existen aquellos que se titulan con una fecha. Ya hemos mencionado el aspecto triple de la estructura que se refleja en las tres secuencias, yo, tú y él.

Cuando se trata de la estructura de la novela La muerte de Artemio Cruz, se plantea otra cuestión interesante, que es el orden de la narración de las unidades marcadas con fechas. Son doce, la fecha o el evento más antiguo es del año 1889 y el más reciente de 1955, sin embargo, no se presentan cronológicamente, sino que comienzan con la fecha de 1941, luego sigue la fecha de 1919, para terminar la novela con el evento del año 1889. Surge la pregunta de si hay algún significado en el orden de representación de estos doce días, o solo la memoria de un hombre a punto de morir dicta este recuerdo aleatorio de ciertos días de su vida. Loveluck (2001: 112) señala la impresión artística del calendario de 
Fuentes de los eventos de la unidad él. Otros críticos ofrecieron diferentes interpretaciones de este orden. González Boixo (2001: 58) divide las fechas en tres grupos, el primer grupo está compuesto de las fechas 1941, 1919, 1913, 1924, 1927, el segundo de 1947, 1915, 1934 y 1939, y el tercero de 1955, 1903 y 1889. Cada grupo comienza con un evento que es similar a la situación actual en la que se encuentra Artemio Cruz, presentada en las secuencias yo y tú que lo preceden. Además, él opina que Fuentes facilita a los lectores la revisión del pasado de Cruz, porque representa personajes y eventos que se mencionan en las unidades yo y tú que preceden (González Boixo 2001: 64). Fuentes, en cualquier caso, influye conscientemente en nuestra percepción y construcción de la imagen de Cruz a través de la presentación de doce días de su vida. De esta manera, vemos el pasado de Cruz, su estatus económico, su posición en la sociedad, su familia, la Revolución Mexicana, pero no podemos hacer un juicio final sobre él, si ha sido corrupto por razones personales o se ha visto obligado a hacerlo debido a las circunstancias, tampoco podemos hacer un juicio sobre sus emociones hacia Catalina, sobre si se trata de un traidor u oportunista (Blanc 1988: 91). Fuentes, con esta estructura, de hecho, ofrece una visión profundamente realista del destino del hombre al reflejar toda su complejidad. Aunque reduce nuestra percepción al mostrar solamente doce días de la vida de Cruz, no simplifica su vida ni insiste en las conclusiones sobre la vida y el ser de Artemio Cruz.

La novela Señas de identidad también tiene una estructura innovadora. Se divide en ocho capítulos, que están simplemente numerados. Igual que Artemio Cruz, Álvaro Mendiola reconstruye su pasado. En la novela aparece el cambio de la segunda y la tercera persona verbal, que es la conexión de distancia y cercanía con las que el personaje principal representa su vida (Stecher 2002: 80):

El desembarco aliado en Normandia, la caída de París, la irrupción del ejército rojo en Polonia y Rumania confirmaban punto por punto los pronósticos pesimistas del tío Eulogio y, hasta el tío César, lector admirado del Mein Kampf y heraldo apasionado de la victoria de los alemanes, parecía humilde y abatido y evocaba con voz lúgubre la eventualidad de un acuerdo con Churchill para frenar el irresistible avance de los rusos. Día tras día, en su mapamundi escolar, Álvaro examinaba acongojado la aterradora mancha roja que extendía ávidamente sus tentáculos sobre la Europa exhausta. El miedo abstracto a la guerra se había transformado poco a poco en cuidado y desvelo respeto a su propio y personal porvenir: ¿qué otra presa podían buscar sino a él, el joven Álvaro, vástago de familia virtuosa y bienpensante, heredero frágil de un mundo delicado y caduco? (Goytisolo 2003: 39) 
Esta dicha cita es un ejemplo de la narración en tercera persona en el primer capítulo, en el que Goytisolo introduce a un narrador omniscente para presentar un evento histórico y mostrar la distancia del protagonista de ese evento mediante la interrupción parcial de una parte del texto escrito en la segunda persona del singular por una narración en la tercera persona.

Si consideramos que la reconstrucción de la vida de Álvaro es el curso principal de la narración, veremos que se trata de fragmentos, porque los eventos no se presentan de forma lineal ni cronológica, sino que se necesita atención para situarlos en el momento al que pertenecen. Dentro del capítulo hay subdivisiones de texto, estas partes no llevan títulos, pero están claramente separadas y representan fragmentos diferentes del curso principal. Los fragmentos no respetan líneas de narración cronológica o causal. Además de esa corriente principal de la narración, de nivel textual primario, aparecen intertextos que se entrelazan con la corriente principal de la narración. Ellos representan la cita de cualquier texto literario o no literario, por ejemplo, el texto periodístico se introduce en el texto primario de la novela con cero o cierto grado de modificación del significado (Stecher 2002: 77). Por ejemplo, todo el último capítulo está compuesto de una sucesión de fragmentos del flujo narrativo principal y el intertexto que habla de la ciudad de Barcelona. Stecher (2002: 85) en su trabajo analiza cómo evolucionan las características estructurales de la novela con la narración de Álvaro, observando cómo con la continuación de los capítulos, los fragmentos con el narrador omnisciente se diluyen, hasta que al final domina la narrativa en la segunda persona, mostrando la cercanía del protagonista a la narración misma, y su ruptura final con la patria española se refleja en la conexión de lírica y prosa que está presente en el último capítulo.

Después de analizar la estructura de la novela Señas de identidad, llegamos a la conclusión de que el escritor español, como el latinoamericano, busca su expresión literaria en el rechazo de las formas antiguas o tradicionales de escribir, y establece la estructura, la forma, la técnica y el lenguaje en el centro de su trabajo. Carlos Fuentes (2013) define una novela como mito, lenguaje y estructura. La estructura innovadora y la experimentación en el campo de la estructura son algunas de las características clave de ambas novelas. Del análisis anterior, se pueden observar muchas similitudes en las estructuras narrativas, de las cuales la fragmentación, la no linealidad, la falta de la secuencia cronológica y causal de los eventos serían las más importantes. 


\section{Técnica narrrativa}

Carlos Fuentes y Juan Goytisolo en sus novelas experimentaron con diversos medios técnicos, por eso a continuación trataremos de mostrar sistemáticamente las técnicas narrativas observadas.

a) Carlos Fuentes aplica la fragmentación en la novela La muerte de Artemio Cruz a través de la estructura fragmentada de la que ya hemos hablado y de la fragmentación de yo de Cruz. El narrador no cambia sustancialmente, yo, tú y él respresentan a Artemio Cruz mismo, además el narrador de cada entidad es Artemio Cruz. El objetivo de Fuentes no es dar una imagen final del mundo, sino ofrecer la presentación de un evento desde diferentes puntos de vista mostrando una visión más real y compleja de la realidad. El mismo rasgo aparece en la novela Señas de identidad y lo hemos discutido en el capítulo anterior.

b) Las prolepsis y analepsis son muy comunes en las novelas. La prolepsis, anticipación, prospectividad o flashforward se refleja en la mención de una historia que sucederá más adelante y que representa el futuro en relación con el momento actual (Prins 2011: 164). La analepsis, retrospección o flashback por otra parte, representa la evocación de uno o más eventos que ocurrieron antes del momento presente o del momento de la narración actual que se interrumpe para abrir espacio a una analepsis (Prins 2011: 19). Las novelas están llenas de analepsis, ya que en una novela el personaje principal está al punto de morir, piensa sobre los eventos que le sucedieron antes, y en la segunda novela, el personaje principal reconstruye su vida. Álvaro usa diferentes materiales en su narración, como álbumes, fotos, películas y estos normalmente introducen las analepsis. La estructura fragmentada en la que los eventos no se presentan cronológicamente permite el surgimiento de numerosas prolepsis y analepsis.

c) La repetición de pronombres, conjunciones, palabras y oraciones también es una técnica narrativa. Como Fuentes utiliza un monólogo, aparecen numerosas repeticiones para representar fielmente el estado del héroe principal. La repetición de la conjunción y es frecuente en la unidad narrativa él para adaptar el texto al estado psíquico de Artemio Cruz (González Boixo 2001: 70). Los pronombres yo y tú se repiten muchas veces, las oraciones se interrumpen, por lo que las partes de las oraciones o las retrospecciones se repiten de nuevo. En esta novela hay una frase repetida muchas veces y se convierte en realidad en el leitmotiv del protagonista, "Esta mañana lo esperaba con alegría. Cruzamos el río a caballo." (Fuentes 2008: 14). Hernán Vidal (1977: 313) piensa que el 
segundo leitmotiv del personaje es la repetición del mandato de abrir las ventanas de la habitación en la que reside: "Abran la ventana" o "Abran las ventanas" (Fuentes 2008: 13, 279) y que enfatiza el deseo de Cruz por la vida, por la supervivencia. La novela Señas de identidad no posee ejemplos de repetición tan llamativos como es el caso de La muerte de Artemio Cruz, ya que no pretende mostrar el estado mental caótico del protagonista. Podemos destacar el ejemplo del último, octavo, capítulo cuando se repite una oración en varios idiomas diferentes, uno de esos ejemplos es el final de la novela:

INTRODUZCA LA MONEDA

INTRODUISEZ LA MONNAIE

INTRODUCE THE COIN

GELDSTUCK EINWARFEN (Goytisolo 2003: 435)

Este procedimiento también está orientado a ajustar el texto al personaje principal, está ubicado en Barcelona, rodeado de turistas, escuchando diferentes idiomas, donde la ciudad misma cambia, adaptándose a otras naciones. Busca su identidad, nació en un país, huyó a otro, un idioma es su idioma materno y está obligado a usar el otro.

d) Ejemplos de oraciones fuera de contexto son numerosos. En la novela La muerte de Artemio Cruz, los leitmotivs que hemos enumerado anteriormente están fuera de contexto. La secuencia yo está formada de tales oraciones, en ella aparecen las oraciones de otros personajes que Cruz escucha y que a veces es muy difícil asociar con el personaje al que pertenecen. Algunas de las oraciones se repiten situadas en el contexto de la unidad él, las podemos ubicar en el tiempo y contexto y luego obtenemos una imagen real de su significado. Tales ejemplos también abundan en la novela Señas de identidad. En el capítulo octavo, ya mencionado, las oraciones de turistas en francés e inglés aparecen fuera de contexto y representan uno de los ejemplos de esta técnica. En este capítulo Álvaro lee un texto sobre la historia de Barcelona, mientras está pensando sobre la ciudad, por lo cual estos comentarios de turistas sobre la ciudad o relacionados con su viaje (on va rater le car / tu te rends compte; come here my darling) son las frases que enfrentan el texto principal y el texto histórico intorducido con la situación real y presente.

e) El uso de signos de puntuación también llama la atención en ambas novelas. El uso excesivo de tres puntos, o puntos y comas, incluso su omisión, se refleja en una serie de ejemplos. Para ilustrar esta técnica citamos un ejemplo interesante de la unidad yo: 
No sé quiénes son... “Cruzamos el río... a caballo"... huelo mi propio aliento... fétido... me recuestan... se abre la puerta... se abren las ventanas... corro... me empujan... veo el cielo... veo las luces borradas que pasan frente a mi vista... toco ... huelo... veo... gusto ... oigo ... me llevan... paso junto... junto... por un corredor... decorado... me llevan... paso junto tocando, oliendo, gustando, viendo, oliendo las tallas suntuosas - las taraceas opulentas - las molduras de yeso y oro - las cajoneras de hueso y carey - las chapas y aldabas - los cofres con cuarterones y bocallaves de hierro los olorosos escaños de ayacahuite - las sillerías de coro - los copetes y faldones barrocos - los respaldos combados - los travesaños torneados - los mascarones policromos - los tachones de bronce - los cueros labrados (Fuentes 2008: 350)

Ejemplos de esta técnica sirven para transmitir un mensaje a través de la estructura de la oración, no solamente a través del contenido, lo que concuerda con la tesis de que el lenguaje, la estructura y la forma son tan importantes como el contenido de la narración. Estos ejemplos se pueden encontrar en toda la obra, sobre todo en las unidades yo y tú. Mientras que en la novela La muerte de Artemio Cruz, la impresión más fuerte la deja el uso excesivo de tres puntos u otros signos de puntuación, lo primero que se puede observar en la novela Señas de identidad es la omisión de signos de puntuación. Al principio de la novela en más de dos páginas, no se utiliza ningún signo de puntuación, excepto un signo que introduce una cita textual. Esta lista caótica de diferentes opiniones, hechos y falsedades acerca de Álvaro Mendiola coincide con la imagen caótica que tiene sobre sí mismo y con la necesidad de conocer su identidad. La técnica de enumeración caótica de datos o descripciones no se usó por primera vez aquí, está presente en otra novela de la literatura española de ese período, en la novela de Luis Martín-Santos de 1961, Tiempo de silencio, por ejemplo en la descripción de Madrid. Además de todo lo anterior, es importante enfatizar que ambas novelas experimentan con el uso de mayúsculas y minúsculas.

f) González Boixo (2001: 72-74) destaca la presentación de escenas simultáneas como una técnica característica de la nueva novela hispanoamericana. La simultaneidad representa una presentación simultánea a través del entrelazamiento de dos o más conjuntos de situaciones y eventos que ocurren al mismo tiempo (Prins 2011: 181). Este procedimiento requiere del lector buena atención y participación activa, pero también permite una visión más amplia de un momento particular. En ambas novelas que son objeto de este análisis, como en muchas otras novelas de literatura contemporánea, hay simultaneidad. 
g) La técnica de collage Goytisolo la utiliza pegando fragmentos diversos que pertenecen a artículos periodísticos, películas, libros, informes y otros materiales. De este modo, Goytisolo presenta varios aspectos de España, personas, paisajes, lugares o eventos históricos. Lo mismo hace Fuentes en el caso de México, y estos diferentes pensamientos, observaciones y actitudes de los héroes principales se agrupan en un collage.

h) Muchas novelas de Carlos Fuentes se caracterizan por la técnica de montaje. Se manifiesta en interrupciones bruscas, transiciones rápidas de escena a escena, flashbacks y montaje (Loveluck 2001: 110). La técnica de montaje denota que el significado de una serie de situaciones o eventos proviene principalmente de la yuxtaposición de estas situaciones y eventos en mayor medida que de sus líneas individuales (Prins 2011: 109). Loveluck (2001: 110-112) con un análisis detallado de la unidad narrativa de 1919 muestra que Fuentes, al cambiar la secuencia causal de los eventos utilizando la técnica de montaje, cambia el curso de la trama y logra el efecto estético deseado y una impresión artística más fuerte.

i) El uso de dos idiomas diferentes se nota en la novela Señas de identidad. Por ejemplo, la narración en el cuarto capítulo está en español y francés. Ambas lenguas las usa Álvaro, español como nativo, francés como residente de Francia, pero en algún caso específico elige otro código lingüístico para mostrar la rebelión contra su idioma nativo. Steacher (2002: 83) piensa que esta aplicación de dos idiomas lleva un mensaje importante, es decir, dado que los lectores de libro que no entiendan francés, no entienden esta parte del texto, significa que es más importante el efecto que produce la introducción de la segunda lengua que el contenido en sí.

Como se puede ver, la mayoría de las técnicas narrativas aplicadas son comunes a ambas novelas, y este hecho habla de la conexión entre la literatura hispánica de los años sesenta del siglo XX a ambos lados del Atlántico y de que los procedimientos narrativos y objetivos de escritura son similares en las obras de Fuentes y Goytisolo.

\section{La temporalidad}

Ya hemos discutido el tema de los tiempos verbales en tres unidades narrativas de la novela La muerte de Artemio Cruz en la sección sobre la estructura narrativa. En cada secuencia domina un tiempo verbal diferente. La unidad yo sigue el estado actual del personaje principal, el flujo de sus pensamientos y está escrito en presente, mientras que la 
unidad él representa la narración de eventos del pasado y está escrito en pasado. La unidad tú no está marcada por la unidad en el uso de los tiempos verbales, aunque prevalece la narración en el futuro. El siguiente ejemplo describe el uso de diferentes tiempos verbales y el uso llamativo de un adverbio que marca el tiempo pasado con el verbo que está en futuro:

Tú, ayer, hiciste lo mismo de todos los días. No sabes si vale la pena recordarlo. Sólo quisieras recordar, recostado allí, en la penumbra de tu recámara, lo que va a suceder: no quieres prever lo que ya sucedió. En tu penumbra, los ojos ven hacia adelante; no saben adivinar el pasado. Sí; ayer volarás desde Hermosillo, ayer nueve de abril de 1959, en el vuelo regular de la Compañía Mexicana de Aviación que saldrá de la capital de Sonora, donde hará un calor infernal, a las 9:55 de la mañana y llegará a México, D.F., a las 16:30 en punto. (Fuentes 2008: 15)

Garrido Domínguez (1996: 202) está de acuerdo con otros teóricos que consideran que el desacuerdo del adverbio con el uso de tiempo verbal representa la separación del lenguaje estándar del literario, es decir, la negación de las reglas del lenguaje durante la escritura de ficción. El uso del tiempo futuro en esta unidad y en el estado concreto de Cruz puede tener el significado de la esperanza y la expectación del futuro (González Boixo 2001: 49).

Cuando se trata del flujo de tiempo en la novela, en trece secuencias que pertenecen a la unidad yo, el tiempo es lineal, va progresivamente, desde el momento en que Cruz se despierta en un estado de salud grave al principio de la novela hasta el final de la novela cuando muere. Desde la primera parte de la unidad tú, descubrimos que la fecha de ayer fue el 9 de abril de 1959, lo que significa que toda la acción que se muestra en la unidad yo se lleva a cabo durante un día, o sea dentro de las doce horas. El número de horas coincide con el número de días de los que Artemio Cruz narra retrospectivamente. Las partes de la unidad él no están cronológicamente narrados y representan un ejemplo de anacronía, o sea de una disonancia entre el orden en el que ocurren los eventos y en el que se narran (Prins 2011: 18). El ya mencionado leitmotiv «cruzamos el río a caballo» se repite muchas veces en la unidad yo, primero fuera de contexto, y luego descubrimos que representa un evento importante de la vida de Cruz relacionado con su hijo Lorenzo. Es un ejemplo de acronía porque carece de cualquier conexión temporal con otros eventos (Prins 2011: 13). Al final, el tiempo en esta novela muestra otra peculiardad que es el 
rasgo cíclico. Fuentes hace una analogía con el entendimiento del tiempo como cíclico de las civilizaciones precolombinas. Además, esta repetición de los mismos ciclos también está presente en la historia moderna de México, por ejemplo, en el hecho de que una oligarquía reemplaza a otra, la historia se muestra así como una repetición constante de los mismos eventos y ciclos (Meyer-Minnemann 2001: 124). Vargas Llosa (1971: 15) señala que las sociedades en las regiones tropicales tienden a glorificar el pasado y mantener ciertas imágenes repetitivas en la memoria colectiva, no solo en los ciclos de tiempo, sino también de una región a otra. Entonces, la historia se repite tanto temporal como espacialmente, y la historia de un país es a menudo la historia de toda la región.

El tiempo verbal dominante en la novela Señas de identidad es el pasado, porque el personaje principal reconstruye su pasado. En la novela también se usa el tiempo presente en aquellas partes que preceden a la introducción de fragmentos que presentan el pasado. No es posible establecer un vínculo en la secuencia de fragmentos en el presente y fragmentos en el pasado, como es el caso en La muerte de Artemio Cruz, porque esta novela, a diferencia de la de Fuentes, no tiene una división en las unidad narrativas caracterizadas por el tiempo verbal que se usa. Sin embargo, la temporalidad tiene características similares. Los eventos se narran retrospectivamente, el anacronismo está presente porque no se respeta la secuencia cronológica de los eventos. Ambas novelas se caracterizan por la experimentación en términos de tiempo, la prioridad se da a la retrospección, en vez de a la narración cronológica lineal.

González Boixo (2001: 46) destaca el aspecto atemporal de la novela La muerte de Artemio Cruz en aquellas partes del monólogo de Cruz en las que se narra sobre los temas importantes de México y la humanidad. Si aplicamos esta teoría en la novela Señas de identidad, veremos que también es posible distinguir el aspecto atemporal en ella, porque se tratan las cuestiones cruciales tanto sobre España, como universales, como es la vida en el exilio.

\section{Narrador y focalización}

Tanto en la novela Señas de identidad como en la novela $L a$ muerte de Artemio Cruz, el personaje principal narra su situación actual o su propio pasado. Se trata de los narradores homodiegéticos, es decir, de los narradores que participan en los eventos narrados (Prins 2011: 69). Esencialmente, aunque la narración no está en primera persona, podemos decir que en las novelas tenemos un ejemplo de narración 
autodiegética, porque el narrador es el protagonista de la novela. Además, queda claro que el protagonista es un narrador, aunque narra sobre sí mismo en otras personas verbales. La novela La muerte de Artemio Cruz posee una unidada narrada en la primera persona singular, en otras dos unidades narrativas el narrador usa los pronombres tú y él, pero continúa narrando sobre sí mismo. En Señas de identidad, no hay narración en primera persona del singular, el narrador normalmente utiliza la segunda persona del singular, la narración en la tercera persona también aparece en ocasiones. A diferencia de la novela La muerte de Artemio Cruz, en la que está completamente claro que Artemio Cruz habla de sí mismo en tercera persona, en la novela Señas de identidad está presente el narrador omnisciente que sirve para narrar ciertas partes de la historia con mayor objetividad. Steacher (2002: 80) señala que existe un caso en la novela cuando el narrador, Álvaro, se presenta en la primera persona del singular, y este se encuentra en el último capítulo cuando se dirige a España:

Oh patria

mi nacimiento entre los tuyos y el hondo amor que

sin pedirlo tú

durante años obstinadamente te he ofrendado (Goytisolo 2003: 433)

Si la tercera persona se usa para distanciar el protagonista de un evento, en este caso la primera persona se usa para expresar el mayor grado de cercanía. Es decir, lo más personal e íntimo para el protagonista es dirigirse a la patria España. El último capítulo representa su ruptura final con la patria y esta es la parte más personal de la novela, que requiere el uso de la primera persona de singular. La busqueda de la identidad en este momento se ha acabado y a pesar del resultado, es el momento de conocer y aceptar su propio ser y su propio yo.

La narración en la segunda persona del singular, está presente en la unidad tú de la novela La muerte de Artemio Cruz y en la mayoría de la narración de la novela Señas de identidad. Estos son casos infrecuentes y raros cuando se trata de la narración, aunque, como lo señala Booth (1976: 168) después de algunas oraciones, el lector se acostumbra a esta forma "artificial" de contar. En la novela de Fuentes, esta unidad no sirve para narrar eventos, sino para expresar las emociones e incertidumbres provocadas por la realidad (Vidal 1976: 306), mientras que en la obra de Goytisolo tú autoreflexivo refleja la influencia de Cervantes, que también está presente en la experimentación con la forma y en las relaciones entre el escritor, personaje y lector (Piras 1999: 168). 
Según la tipología de Booth (1976: 167-186), los narradores en estas dos novelas son dramatizados, es decir, están caracterizados como personas. Participan en la acción y son conscientes de sí mismos, porque son conscientes de narrar, o reflexionar sobre, una obra literaria. Nos permiten conocer a otros personajes, sobre todo a ellos mismos.

Enfoque, punto de vista o perspectiva es la posición observacional o conceptual en relación con la cual se presentan las situaciones y los eventos de la narración (Prins 2011: 196). En cuanto a la posición en relación con la historia, Rimmon-Kenan (2006: 44) distingue la focalización externa e interna, y en el caso de ambas novelas, prevalece el enfoque interno, ya que el enfoque está "dentro de los eventos presentados". Sin embargo, cuando Artemio Cruz en la unidad él se refiere retrospectivamente a ciertos días de su vida, narrándolos en la tercera persona del singular, observa en el momento del habla y no en el momento de la acción (Rimon Kenan 2006: 44), entonces la focalización cambia a la externa. El ejemplo más claro se encuentra en el último evento dela unindad narrativa él que sucede el 9 de abril de 1889. Artemio narra sobre su nacimiento. Está claro que Artemio aún no nacido no habla de sí mismo en el momento en que se encuentra en el centro del útero de su madre, es el Artemio más maduro el que cuenta el evento. La focalización en la novela Señas de identidad es similar a la de La muerte de Artemio Cruz. Álvaro es personaje-focalizador, es decir, la focalización es interna, excepto en fragmentos cuando aparece el narrador omnisciente y cuando está presente la focalización externa. El objeto de focalización también puede ser visto desde la posición externa o interna y no tiene que coincidir con el tipo de focalización en relación con la historia (Rimon Kenan 2006: 44). Dependiendo del objeto o la persona que se enfoca, en las novelas tenemos ejemplos de ambos tipos, por un lado, algunos personajes se representan solo a través de la caracterización de rasgos externos, pero por otro, a veces se presentan los pensamientos y sentimientos de los personajes. De hecho, la focalización no es fija, sino variable. Artemio Cruz mismo, presentado desde varias perspectivas diferentes, es la representación de un ser real y la realidad mexicana, sin un mensaje ideológico y juicios finales (Blanc 1988: 93). Álvaro Mendiola, también, está presentado desde diferentes perspectivas, pero al final forma una actitud clara sobre España. En consecuencia, podemos concluir que cuando se trata del aspecto ideológico, un tono más neutro se encuentra en La muerte de Artemio Cruz. 


\section{Conclusión}

Según Carlos Fuentes (2013), crear un lenguaje para los latinoamericanos significa crear un ser, una identidad. Goytisolo, por otra parte, destaca que la tarea más importante de la novela española es destruir el lenguaje antiguo y producir el nuevo a través de la novela, de este modo, él como Cervantes, convirtió el lenguaje en desafío e investigación (Fuentes 2013).

Para ambos escritores, la novela es el área donde se examinan las posibilidades del lenguaje y la creatividad artística. Experimentar con técnicas narrativas, estructura y lenguaje es la tarea más importante del autor, no para la experimentación en sí, sino para que la estructura y el lenguaje se ajusten a la historia y los personajes. El mensaje no está en la narración, en las palabras tampoco, sino en la suma de todos los procedimientos narrativos. Lo dicho significa que cuando se busca la esencia de España o México, se realiza usando todos los medios disponibles, no solamente a través de palabras o historia. Después de un análisis comparativo de las novelas La muerte de Artemio Cruz y Señas de identidad, queda claro por qué Fuentes y Goytisolo se apreciaron y entablaron amistad, lo que representa una reunión importante para la literatura española e hispanoamericana. Aunque los contextos son diferentes, Fuentes y Goytisolo determinan problemas de la novela de mediados del siglo XX de la misma manera, tratan sus historias y novelas de la misma manera y, finalmente, de la misma manera perciben la identidad de un individuo y una nación. Por lo tanto, hay muchas similitudes en los procedimientos narrativos de las dos novelas analizadas. 


\section{BIBLIOGRAFÍA}

Blanc, Mario A. "La complejidad apasionante de La muerte de artemio Cruz". La Palabra y el Hombre, Núm. 67 (1988): 83-93. Web. 01 Sep. 2018. But, Vejn. Retorika proze. Beograd: Nolit, 1976. Štampano.

Fuentes, Carlos. La muerte de Artemio Cruz. Madird: Alfaguara, 2008. Impreso.

Fuentes, Carlos. La nueva novela hispanoamericana. Pamplona: Leer-e, 2013. Web. 01 Sep. 2019.

Garrido Domínguez, Antonio. El texto narrativo. Madrid: Editorial Síntesis, 1996. Impreso.

Goytisolo, Juan. Señas de identidad, Madrid: Alianza Editorial, 2003. Impreso.

Goytisolo, Juan. "Semblanza de nacionalidad cervantina". Claves de la Razón Práctica, Núm. 217 (2011): 46-47. Web. 31 Ago. 2019.

González Boixo, José Carlos. "Imaginar el pasado, recordar el futuro". Carlos Fuentes. La muerte de Artemio Cruz, Madrid: Cátedra, 2001: 11-99. Impreso.

Loveluck, Juan. "Intención y forma en La muerte de Artemio Cruz". Georgina García-Gutiérrez. Carlos Fuentes desde la crítica. México: UNAM, 2001: 103-118. Google Books. Web. 01 Sep. 2018.

Meyer-Minnemann, Klaus. "La muerte de Artemio Cruz: tiempo cíclico e historia del México moderno". Georgina García-Gutiérrez. Carlos Fuentes desde la crítica. México: UNAM, 2001: 119-130. Google Books. Web. 01 Sep. 2018.

Piras, Pina Rosa. "El cervantismo de Juan Goytisolo". Cervantes: Bulletin of the Cervantes Society of America. Vol. 19, Núm. 2 (1999): 167-179. Web. 02 Sep. 2018.

Prins, Džerald. Naratološki rečnik. Beograd: Službeni glasnik, 2011. Štampano.

Rimon-Kenan, Šlomit. Narativna proza. Beograd: Narodna knjiga - Alfa, 2006. Štampano.

Stecher, Lucia. "Estructura y lenguaje en Señas de identidad de Juan Goytisolo". Revista Chilena de Literatura. Núm. 60 (2002): 67-86. Web. 01 Sep. 2018.

Vargas Llosa, Mario. García Márquez: historia de un deicidio. Barcelona: Barral Editores, 1971. Impreso.

Vidal, Hernán. "El modo narrativo en La muerte de Artemio Cruz de Carlos Fuentes". Thesaurus, Vol. 31, Núm. 2 (1977): 300-326. Web. 01 Sep. 2018. 


\title{
NARRATIVE PROCEDURES OF CARLOS FUENTES AND JUAN GOYTISOLO
}

\begin{abstract}
Summary
In this paper, we analyse the narrative procedures in the novels The Death of Artemo Cruz of Carlos Fuentes and Signs of Identity of Juan Goytisolo. The comparison of Fuentes and Goytisolo's narrative methods is done through the analysis of narrative structure of the novels, narrative techniques, temporality, narrator and focalisation. These aspects of the novel are highlighted because they are crucial when it comes to narration, and both authors have shown that the structure, language and technique are equally important as the story itself. Since this is a comparative topic, we seek and describe the common characteristics of narrative procedures, but we also try to point out the differences. We note that the perception of the novel and the role of the writer, as well as the writing strategy of Fuentes and Goytisolo, are similar, which represents an important meeting of the Spanish and Hispanic novels in the sixties of the twentieth century. Although it would be possible to find even more characteristics to deal with and more examples, we retain on the analysis of the most striking features in order to illustrate the phenomena that we primarily describe or define theoretically.

Keywords: Carlos Fuentes, Juan Goytisolo, narrative procedure, novel,
\end{abstract} narration. 